\title{
Video Article \\ Autologous Endothelial Progenitor Cell-Seeding Technology and Biocompatibility Testing For Cardiovascular Devices in Large Animal Model
}

\author{
Alexandra E. Jantzen ${ }^{1}$, Whitney O. Lane ${ }^{2}$, Shawn M. Gage ${ }^{3}$, Justin M. Haseltine ${ }^{1}$, Lauren J. Galinat ${ }^{1}$, Ryan M. Jamiolkowski ${ }^{4}$, Fu-Hsiung Lin ${ }^{3}$, \\ George A. Truskey ${ }^{1}$, Hardean E. Achneck ${ }^{3}$ \\ ${ }^{1}$ Department of Biomedical Engineering, Duke University \\ ${ }^{2}$ School of Medicine, Duke University \\ ${ }^{3}$ Department of Surgery, Duke University Medical Center \\ ${ }^{4}$ School of Medicine, University of Pennsylvania
}

Correspondence to: Hardean E. Achneck at hardean.achneck@duke.edu

URL: https://www.jove.com/video/3197

DOI: doi:10.3791/3197

Keywords: Bioengineering, Issue 55, Stent, Titanium, Thrombosis, Endothelial Progenitor Cell, Endothelium, Biomaterial, Biocompatibility, Bioengineering, Translational Medicine, Vascular Surgery, Porcine

Date Published: 9/9/2011

Citation: Jantzen, A.E., Lane, W.O., Gage, S.M., Haseltine, J.M., Galinat, L.J., Jamiolkowski, R.M., Lin, F.H., Truskey, G.A., Achneck, H.E. Autologous Endothelial Progenitor Cell-Seeding Technology and Biocompatibility Testing For Cardiovascular Devices in Large Animal Model. J. Vis. Exp. (55), e3197, doi:10.3791/3197 (2011).

\section{Abstract}

Implantable cardiovascular devices are manufactured from artificial materials (e.g. titanium (Ti), expanded polytetrafluoroethylene), which pose the risk of thromboemboli formation ${ }^{1,2,3}$. We have developed a method to line the inside surface of Ti tubes with autologous blood-derived human or porcine endothelial progenitor cells (EPCs) ${ }^{4}$. By implanting Ti tubes containing a confluent layer of porcine EPCs in the inferior vena cava (IVC) of pigs, we tested the improved biocompatibility of the cell-seeded surface in the prothrombotic environment of a large animal model and compared it to unmodified bare metal surfaces ${ }^{5,6,7}$ (Figure 1). This method can be used to endothelialize devices within minutes of implantation and test their antithrombotic function in vivo.

Peripheral blood was obtained from $50 \mathrm{~kg}$ Yorkshire swine and its mononuclear cell fraction cultured to isolate EPCs ${ }^{4,8}$. Ti tubes (9.4 mm ID) were pre-cut into three $4.5 \mathrm{~cm}$ longitudinal sections and reassembled with heat-shrink tubing. A seeding device was built, which allows for slow rotation of the Ti tubes.

We performed a laparotomy on the pigs and externalized the intestine and urinary bladder. Sharp and blunt dissection was used to skeletonize the IVC from its bifurcation distal to the right renal artery proximal. The Ti tubes were then filled with fluorescently-labeled autologous EPC suspension and rotated at $10 \mathrm{RPH} \times 30 \mathrm{~min}$ to achieve uniform cell-coating ${ }^{9}$. After administration of $100 \mathrm{USP} / \mathrm{kg}$ heparin, both ends of the IVC and a lumbar vein were clamped. A $4 \mathrm{~cm}$ veinotomy was performed and the device inserted and filled with phosphate-buffered saline. As the veinotomy was closed with a 4-0 Prolene running suture, one clamp was removed to de-air the IVC. At the end of the procedure, the fascia was approximated with 0-PDS (polydioxanone suture), the subcutaneous space closed with 2-0 Vicryl and the skin stapled closed.

After 3 - 21 days, pigs were euthanized, the device explanted en-block and fixed. The Ti tubes were disassembled and the inner surfaces imaged with a fluorescent microscope.

We found that the bare metal Ti tubes fully occluded whereas the EPC-seeded tubes remained patent. Further, we were able to demonstrate a confluent layer of EPCs on the inside blood-contacting surface.

Concluding, our technology can be used to endothelialize Ti tubes within minutes of implantation with autologous EPCs to prevent thrombosis of the device. Our surgical method allows for testing the improved biocompatibility of such modified devices with minimal blood loss and EPCseeded surface disruption.

\section{Video Link}

The video component of this article can be found at https://www.jove.com/video/3197/

Protocol

\section{Endothelial progenitor cell isolation}

1. Thirty days prior to EPC-seeded tube implantation, prepare a $60 \mathrm{ml}$ syringe with $15 \mathrm{ml}$ of anticoagulant citrate dextrose solution and secure with a 3-way stop cock for EPC isolation from peripheral pig blood. 24 hours prior to the blood draw, precoat two 12-well plates with Type 1 rat collagen $(50 \mu \mathrm{g} / \mathrm{ml} \text {, dissolved in } 0.02 \mathrm{~N} \text { acetic acid solution })^{4}$. 
2. Conduct all animal care and experimentation in accordance with the National Institute of Health Guidelines for the Care and Use of Laboratory Animals and only after approval of overseeing Institutional Animal Care and Use Committee.

3. Sedate a female Yorkshire pig $(45 \mathrm{~kg})$ with Acepromazine $(1.1 \mathrm{mg} / \mathrm{kg})$ and Ketamine $(22 \mathrm{mg} / \mathrm{kg})$ intramuscular via a $19 \mathrm{G}$ butterfly needle.

4. Intubate the pig with an endotracheal tube (30 cm length, $8 \mathrm{~mm} \mathrm{ID})$ and anesthetize pig with Isoflurane ( $4.5 \%$ of tidal volume by mask).

5. Monitor the pig during the procedure by measuring oxygen saturation, heart rate and temperature. Maintain thermoregulation by using an automated heated operating table and heating blanket and by warming the infused fluids.

6. Place the pig in the supine position on the operating table, secure its hind limbs caudolaterally, and clean with Chlorhexidine followed by DuraPrep sterilization. Then proceed with draping the pelvic area.

7. Insert a $21 \mathrm{G} \times 7 \mathrm{~cm}$ needle from a $5 \mathrm{~F}$ micro-introducer kit just medial to a palpable femoral pulse (medial to the vastus medialis and lateral to the gracilis muscle) into the femoral vein and cannulate the vein using Seldinger technique. Connect the prepared $60 \mathrm{ml}$ syringe to the intravascular catheter and draw $45 \mathrm{ml}$ of blood.

8. Hold pressure on the vessel puncture site to achieve hemostasis $(5 \mathrm{~min})$, discontinue the anesthesia and recover the pig. The animal is monitored until full recovery and returned to its cage.

9. Dilute the blood solution 1:1 with Hank's buffered salt solution (without $\mathrm{CaCl}_{2}, \mathrm{MgCl}_{2}, \mathrm{MgSO}_{4}$ ) and layer on equal volumes of Histopaque to create well-defined layers. Centrifuge (30 min, $740 \mathrm{~g}$, low break setting) and collect mononuclear cell (MNC) layer. Resuspend and wash MNCs $\times 3$ with Dulbecco's Phosphate Buffered Saline (DPBS) (10 min, $515 \mathrm{~g})$ before plating into two 12-well plates in full growth medium (EBM-2 medium with $2 \%$ Porcine Serum (PS) and EGM-2 SingleQuots) at $37^{\circ} \mathrm{C}, 5 \% \mathrm{CO}_{2}$

10. Slowly change medium every 24 hours for the first 7 days, then every other day. Identify EPC colonies after an average time of 7 days (Figure 2).

11. Expand EPCs in culture once they cover $1 / 4$ of the 12 -well surface area. Confirm EPC identity with flow cytometry by testing for presence of surface markers CD31 and absence of CD14, CD45. Other assays that may be performed include cell morphology and nitric oxide III activity after exposure to flow ${ }^{4}$.

\section{Titanium tube assembly}

1. Section a Ti tube longitudinally into 3 equal 120 -degree units $(4.5 \mathrm{~cm}$ long) with a 72 teeth HHS slitting saw held in place by a saw arbor in a vertical milling machine. Run it at 300 RPM and keep the cutting area saturated with Tap Magic Cutting Fluid at all times during the cut (Figure 3).

2. Polish the inner surface with a bench grinder and a Scotch-Brite metalworking wheel. Then manually polish with $3 \mathrm{M}$ emery cloth to further smooth and even surface to remove any visible pits.

3. Clean the Ti pieces with Alconox soap solution, followed by 5 min submersion in aqua regia (1:3 concentrated nitric acid to concentrated hydrochloric acid), followed by rinsing with several liters of water ${ }^{10}$. Exercise extreme caution as aqua regia is highly corrosive and potentially explosive!

4. Sonicate Ti sections $\times 16$ min in Alconox soap solution, rise $\times 30$ in deionized water and sonicate again $\times 16$ min in deionized water. Allow to dry in laminar flow hood.

5. Cut PVC heat-shrink tubing to $4.5 \mathrm{~cm}$ length and thoroughly clean per $\mathbf{2 . 4}$.

6. Use tweezers to place the 3 cleaned Ti sections onto a supportive mandrel (machined from aluminum, $8.5 \mathrm{~mm}$ OD) and place sleeve of PVC heat-shrink tubing around Ti sections (Figure 4). Shrink tubing with a heat gun while turning mandrel to evenly wrap Ti sections tightly together.

\section{Seeding device and component assembly}

1. Cut silicone tubing to a length of $2.5 \mathrm{~cm}$ and $3.5 \mathrm{~cm}$.

2. Section $5 \mathrm{cc}$ plastic syringe at the $0.8 \mathrm{ml}$ mark and keep 'head piece' with luer end.

3. Thoroughly clean $2 \mathrm{x}$ silicone sections, syringe 'head piece' and a luer cap by sonication as described under 2.4 .

4. Add silicone tubing to each end of assembled Ti tube from 2.5, extending by $5 \mathrm{~mm}$ over PVC tubing.

5. Insert cut end of syringe 'head piece' into short silicone tubing, so that it abuts Ti tube (Figure 5).

6. Seal finished Ti tube assembly with one luer cap in Tyvek pouch and gas-sterilize with ethylene oxide $\left(18\right.$ hours at $\left.55^{\circ} \mathrm{C}\right)$.

7. Mount a synchronous timing motor $(10 \mathrm{RPH})$ onto a plexiglass platform and connect its axle to a syringe holder (machined out of aluminum, fits $5 \mathrm{cc}$ syringe) (Figure 6).

\section{EPC-seeding of Ti tube inner surface}

1. Expand EPCs as isolated under 1. to 3 confluent T-75 flasks (or at least $9 \times 10^{6}$ cells).

2. On the day of surgery, fluorescently label cells with long-term dye $(\mathrm{PKH} 26)^{11}$. Begin by rinsing cultured EPCs twice in serum free medium. Caution to limit light exposure to protect cells during and after labeling.

3. Cover cells with $4 \mu \mathrm{M}$ PKH26 in Diluent $\mathrm{C}$ (dye solution) at room temperature for 4 minutes.

4. Stop labeling reaction by adding porcine serum in equal volume to dye solution. One min later, dilute combined solution $1: 1$ with full growth medium.

5. Aspirate liquid and rinse cells $\times 3$ with full growth medium.

6. Wash EPCs twice in DPBS (without $\mathrm{CaCl}_{2}, \mathrm{MgCl}_{2}$ ).

7. Cover cells in trypsin and incubate at $37^{\circ} \mathrm{C}$ for 3 minutes and confirm detachment under a light microscope. Add Trypsin Neutralization Solution in double the volume of trypsin used.

8. Combine cell solutions into a single tube and mix by pipetting. Add $10 \mu \mathrm{l}$ of cell solution into each side of a hemocytometer for counting.

9. Centrifuge cell solution (1500 RPH, $5 \mathrm{~min}$.). Count cells and resuspend pellet at $2-2.5 \times 10^{6} \mathrm{EPCs} / \mathrm{ml}$ in serum free medium. Note the minimum volume to fill the tube assembly is $4.5 \mathrm{ml}$.

10. Lay out sterile towel in biological hood for sterile field. Open gas-sterilized Ti tube assembly and extra 5 cc syringe onto sterile field.

11. With sterile gloves, remove plunger from $5 \mathrm{cc}$ syringe and keep on field for later use. Affix luer cap to this syringe. 
12. Pipet the 4.5 - $5 \mathrm{ml}$ EPC suspension under 4.9 into the open syringe barrel. Insert plunger securely back into open end of syringe.

13. Hold syringe with cap upward and remove cap. Insert syringe with luer end first into open silicone tubing of Ti tube assembly until snug; do not advance within Ti tube.

14. Advance syringe plunger slowly until cell solution reaches top of cut syringe 'head piece,' removing bubbles from system. Close with luer cap and insert entire assembly into sterile sheath, sealing open end with tape.

15. Insert this entire assembly into machined syringe holder of 3.7. Place in incubator at $37^{\circ} \mathrm{C}$ and adjust platform so that Ti tube portion of seeding chamber is level, using water level gauge (Figure 6).

16. Allow Ti tube assembly to rotate 30 minutes before implantation.

\section{Implantation of Ti tube into porcine inferior vena cava}

1. Twenty-four hours prior to surgery, premedicate the pig (from which EPCs were isolated) with a Fentanyl patch (100 $\mu \mathrm{g} / \mathrm{hr}$ transdermal; keep patch in place $\times 72$ hours).

2. Keep pig NPO overnight and administer Baytril (Enrofloxacin) preoperatively at the day of surgery (5 mg/ $\mathrm{kg}$, IM) and for 7 days following, every 24 hours as antibiotic prophylaxis.

3. Sedate, intubate and anesthetize the pig as described under 1.3 (tidal volume of $10-15 \mathrm{ml} / \mathrm{kg}$ ) and secure the pig in supine position on the operating room table. Monitor the pig during the procedure by measuring oxygen saturation, heart rate and temperature. Maintain thermoregulation by using an automated heated operating table and heating blanket and by warming the infused fluids.

4. Insert an $18 \mathrm{G}$ IV catheter into the pig's ear vein and protect pig's eyes with Vetropolycin eye drops.

5. Clean, prep and drape the pig's abdomen as in 1.6. Incise the midline with a \# 15 scalpel blade from the 2 nd set of mammary glands cranially to the 2 nd to last set caudally.

6. Carry the dissection down to the abdominal fascia with electrocautery

7. Lift the peritoneum with Mosquito forceps, and carefully enter it with Metzenbaum scissors.

8. Externalize the urinary bladder and place a 3-O Vicryl purse-string suture into the bladder wall. Place a stab incision in its middle and insert a $16 \mathrm{~F}$ Foley catheter. Administer intravenous fluids (Lactate Ringers) to titrate the urine output to $>/=1 \mathrm{ml} / \mathrm{kg} / \mathrm{hr}$ during the surgery.

9. Following, externalize the small and large bowel and place two Balfour surgical retractors to expose the posterior aspect of the peritoneal cavity and identify the inferior vena cava (IVC).

10. Using sharp and blunt dissection, carefully free the IVC from surrounding tissue and skeletonize the vessel from the right renal artery proximal to the bifurcation of the IVC distal. Exercise extreme care during the IVC dissection as even a very small defect in the IVC may lead to rapid hemorrhage and exsanguination of the animal.

11. Ligate all side branches of the IVC segment to ensure that there will be no bleeding around the Ti tube to be implanted. Note the usually large two posterior lumbar veins that require very careful dissection and ligation. Further, note that immediately proximal to the bifurcation of the IVC, a large lumbar vein is commonly encountered on the posteromedial side and must be dissected free and controlled with vessel loops in preparation for clamp placement.

12. Proceed with seeding the Ti tube simultaneously as outlined under 4.

13. Administer $100 \mathrm{USP} / \mathrm{kg}$ of heparin immediately prior to clamping the IVC. Place 45 degree angle surgical clamps distally on the IVC and lumbar vein, and then proximally.

14. Create a longitudinal veinotomy $(4 \mathrm{~cm})$ between the proximal and distal clamps using a \# 11 scalpel blade followed by extension with Potts scissors.

15. Evacuate any blood from the IVC and flush its inner lumen with sterile DPBS.

16. Now insert the EPC-seeded Ti tube (or a bare metal control) into the IVC and fill it with DPBS (with $\mathrm{CaCl}_{2}, \mathrm{MgCl}_{2}$ ) to prevent the cells from drying and to evacuate air.

17. Close the veinotomy with a 4-0 Prolene running suture and remove one proximal clamp to de-air the IVC through a small amount of backbleeding. Place a 'stay-suture' through the vein wall into the PVC tubing to prevent migration of implant over time.

18. Close the fascia with O-PDS on a CT needle and the subcutaneous space with 2-O Vicryl (running sutures). Close the skin with staples.

19. Administer up to $20 \mathrm{ml}$ of $0.25 \%$ Marcaine (Bupivacaine) subcutaneously along the incision site and cover the wound with gauze and Tegaderm. Also give Flunixin (2.2 mg/ kg Q $24 \mathrm{hr})$ and Oxymorphone $(0.15 \mathrm{mg} / \mathrm{kg} \mathrm{Q} 3-4 \mathrm{hr})$ subcutaneously as needed for pain.

20. Discontinue anesthesia, monitor the pig until awake and return to cage. Monitor pig twice daily for signs of distress / pain, standing and ambulation, stool and urine output, and skin color indicating normal perfusion.

\section{Explantation of Ti tube}

1. After 3 weeks, sedate, intubate and anesthetize the pig as described in $1.3 \mathbf{- 1 . 4}$.

2. Proceed with a laparotomy as described in $\mathbf{5 . 5} \mathbf{- 5 . 8}$.

3. Note that scarring will conceal the implant site but you can palpate the rigid Ti tube in situ.

4. Dissect out the IVC as described in 5.9 - 5.11 and explant the Ti tube en-block with the surrounding IVC with heavy scissors.

5. Euthanize the animal with Euthasol euthanasia solution $(390 \mathrm{mg} / \mathrm{ml}$ Pentobarbital Sodium and $50 \mathrm{mg} / \mathrm{ml}$ Phenytoin Sodium at $1 \mathrm{ml} / 10 \mathrm{lbs})$.

\section{Fixation and imaging Ti inner surface}

1. Rinse the excised vein segment with Ti tube in DPBS solution and photograph its lumen (patent or occluded) with a high resolution digital camera (Figure 7).

2. Following, fix the specimen by submersion in $3.7 \%$ paraformaldehyde for a minimum of 15 minutes. Rinse specimen in DPBS solution

3. Very carefully incise the surrounding vein and PVC tubing to open the Ti tube. Place the 3 sections under a fluorescent microscope with the inside surfaces facing the objective/ light source and image under $550 \mathrm{~nm}$ excitation wavelength to visualize PKH26-labeled EPCs in red/ orange color (Figure 8A). If desired, cells can be further stained (e.g. Platelet Endothelial Cell Adhesion Marker (PECAM)-stain to visualize cell borders (Figure 8B), DAPI-stain to visualize nuclei, etc.). 


\section{Representative results:}

Following execution of this protocol, physicians and scientists are able to endothelialize solid tube structures with autologous blood-derived endothelial progenitor cells in a large animal model. Figure $\mathbf{2}$ shows that EPCs isolated with our method appear as colonies with cobblestone morphology after approximately 7 days in culture. Our seeding device illustrated in Figures $\mathbf{5}$ and $\mathbf{6}$ allows for slow rotation of Ti tubes filled with the EPC suspension and results in uniform coverage of the tube's inner surface under sterile conditions ${ }^{9}$.

Our implantation surgery allows for testing the propensity of biomaterials, such as Ti, for thrombosis in a large animal model. We found that pigs tolerate this procedure well and that this implantation can be achieved with only minimal blood loss and without EPC layer disruption.

Figure 7 shows that a bare Ti tube completely occludes, whereas our EPC-lined tube remains patent even in the prothrombotic low shear environment of the inferior vena cava. Further, the presence of a confluent layer of fluorescently-labeled cells confirms the success of this method as shown in Figure 8.

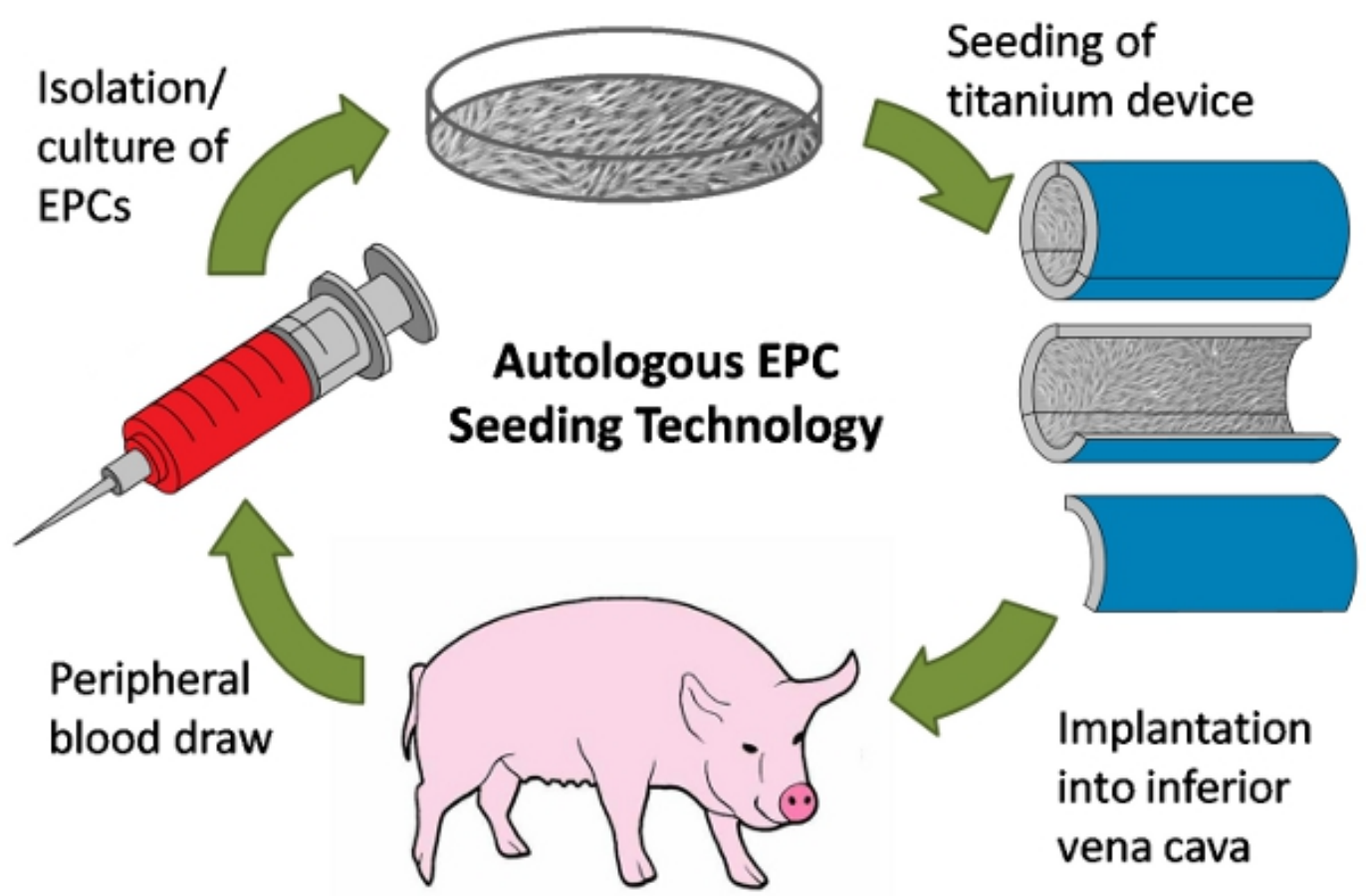

Figure 1. Schematic of autologous endothelial progenitor cell (EPC) seeding experiment. First, peripheral blood is drawn from a pig. Next, EPCs are isolated from the blood and expanded in culture. EPCs are then used to line a titanium (Ti) tube device, which is then surgically implanted into the inferior vena cava of the same pig from which cells were isolated. 


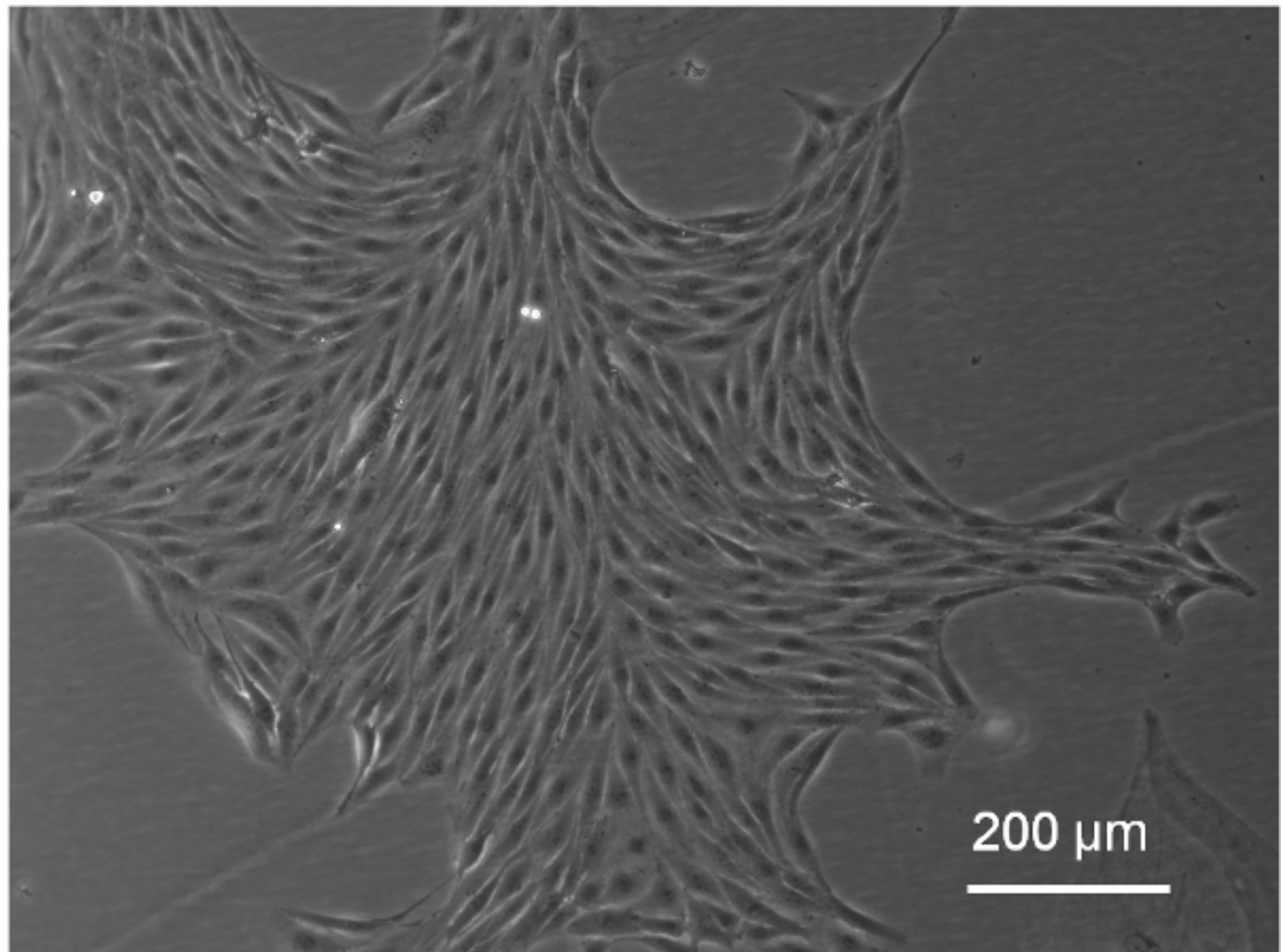

Figure 2. Representative colony of EPCs in culture, approximately 7 days following isolation procedure (imaged with an inverted Leica DMIL microscope with Imaging camera and QCapture software).

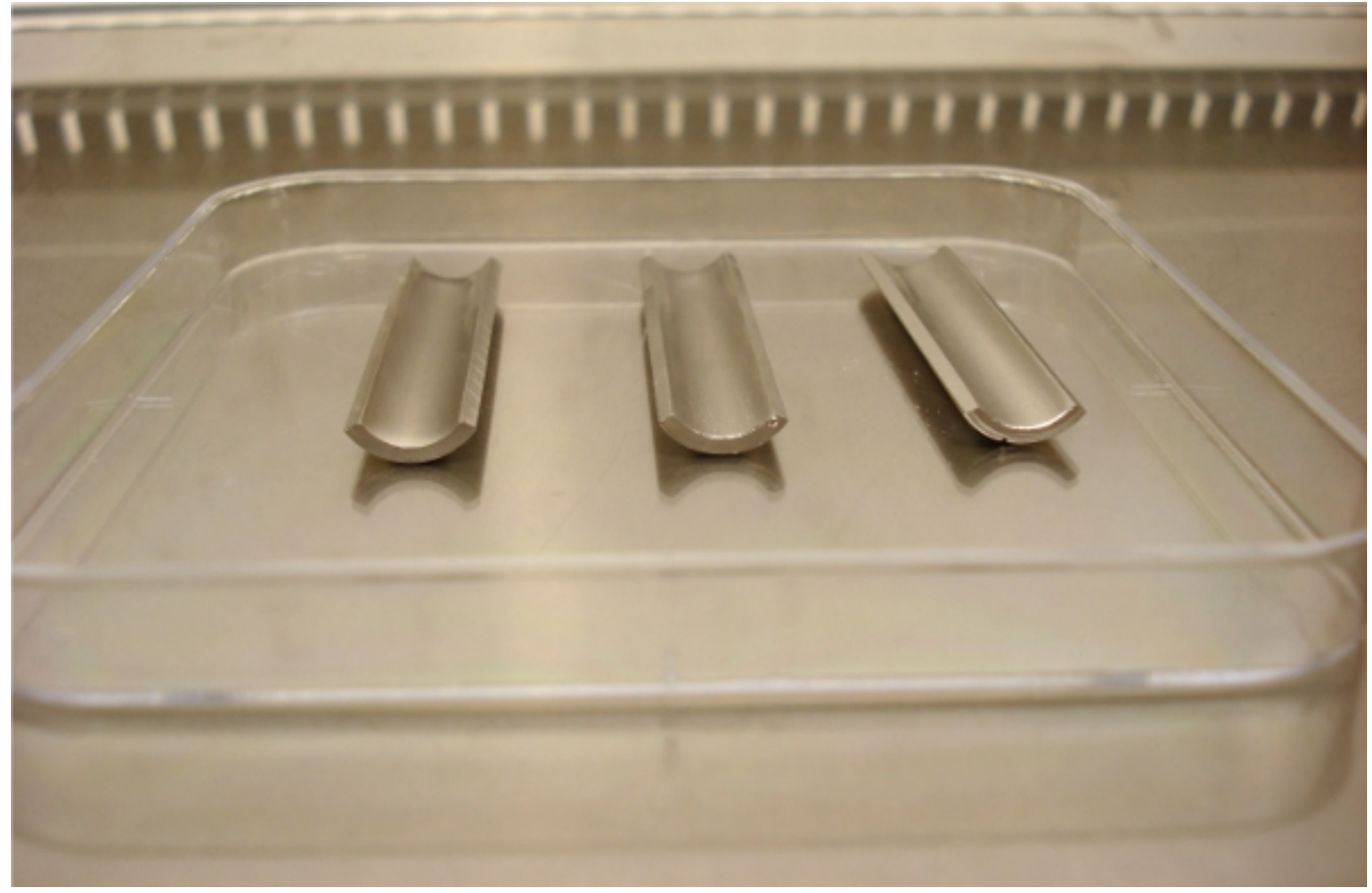

Figure 3. Ti tube sections prior to assembly. Ti tubing is cut into 3 equal sections longitudinally, and then cut to $4.5 \mathrm{~cm}$ length. Inner surfaces of Ti sections are polished using a bench grinder and emery cloth to remove visible pits. 


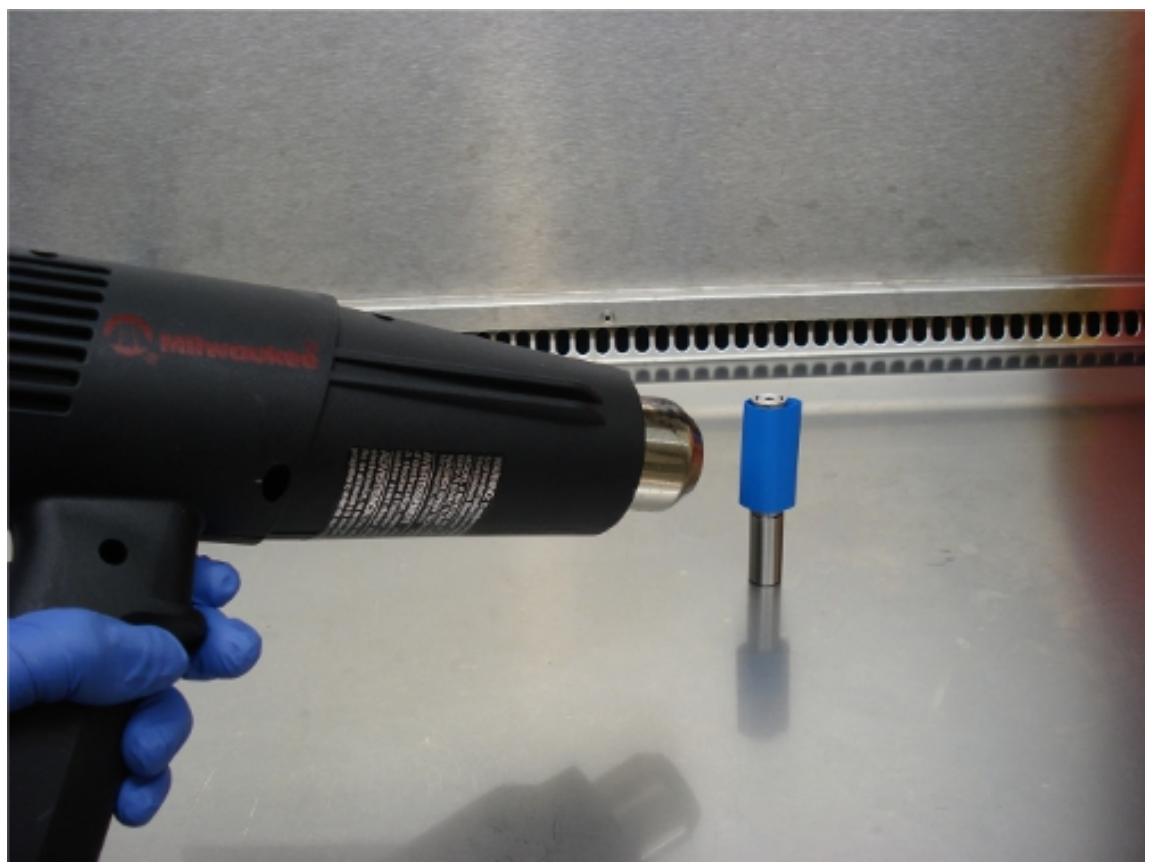

Figure 4. Assembly of Ti tube sections with PVC heat-shrink tubing (blue) and heat gun. Ti sections are supported on a machined aluminum mandrel which extends through Ti sections and matches the dimensions of the Ti tube inner diameter.

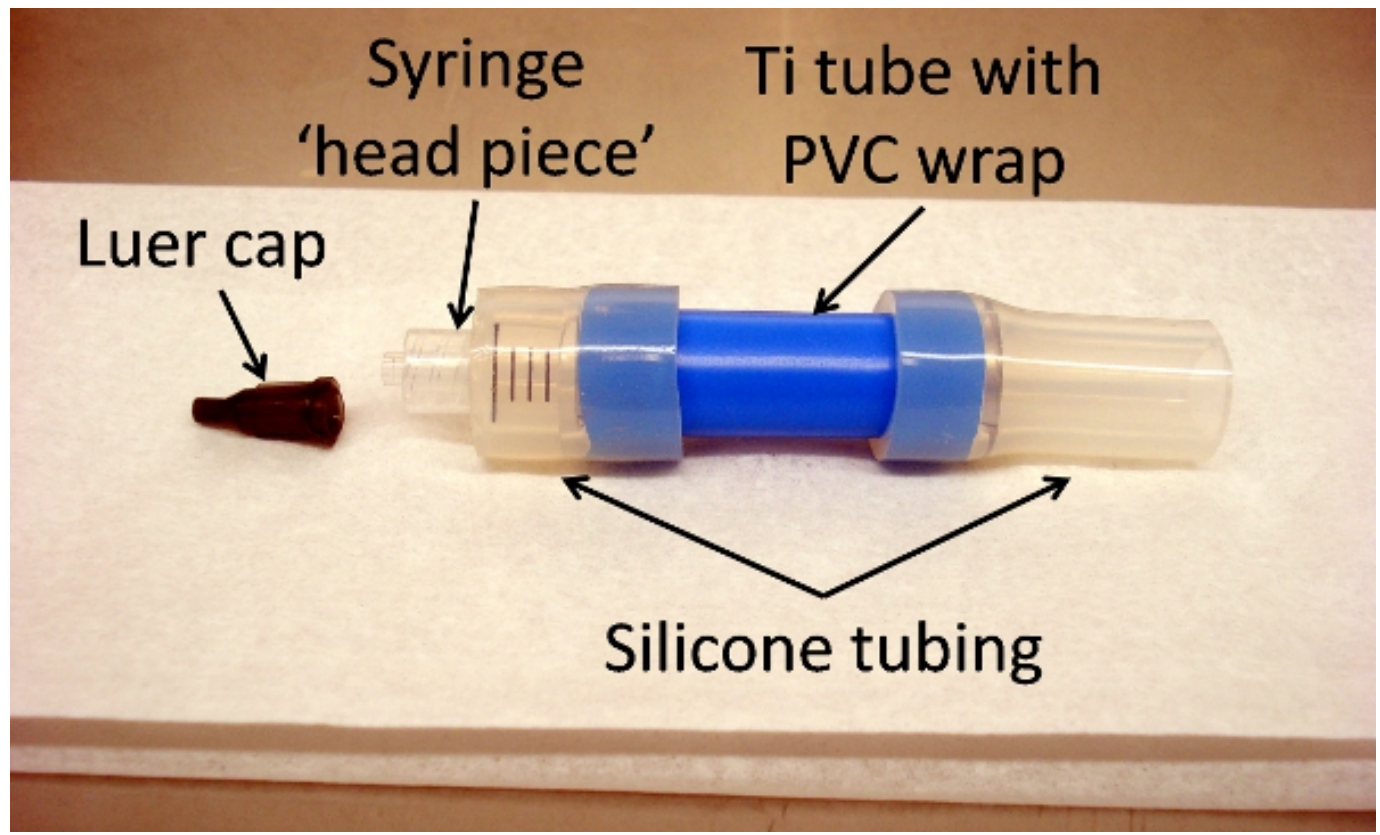

Figure 5. Titanium tube assembly, showing all components: luer cap, syringe 'head piece,' silicone tubing, and Ti tube with PVC wrap (blue). Assembly is put together prior to sterilization for surgical use. 


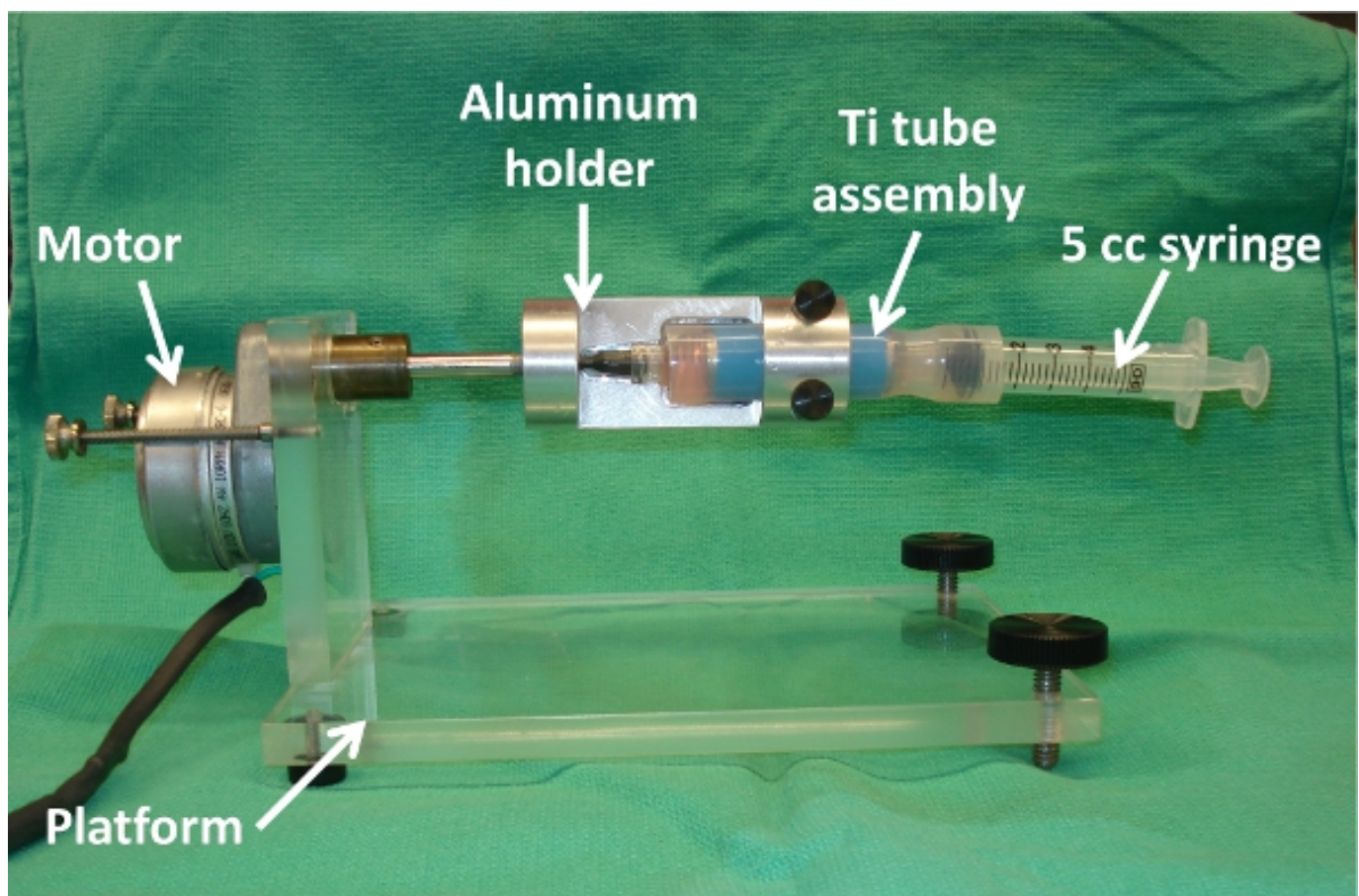

Figure 6. Ti tube seeding setup inside incubator, showing motor, platform, machined aluminum syringe holder, Ti tube assembly, and $5 \mathrm{cc}$ syringe. Note: Assembly is shown without protective sterile sheath for visualization purposes. 

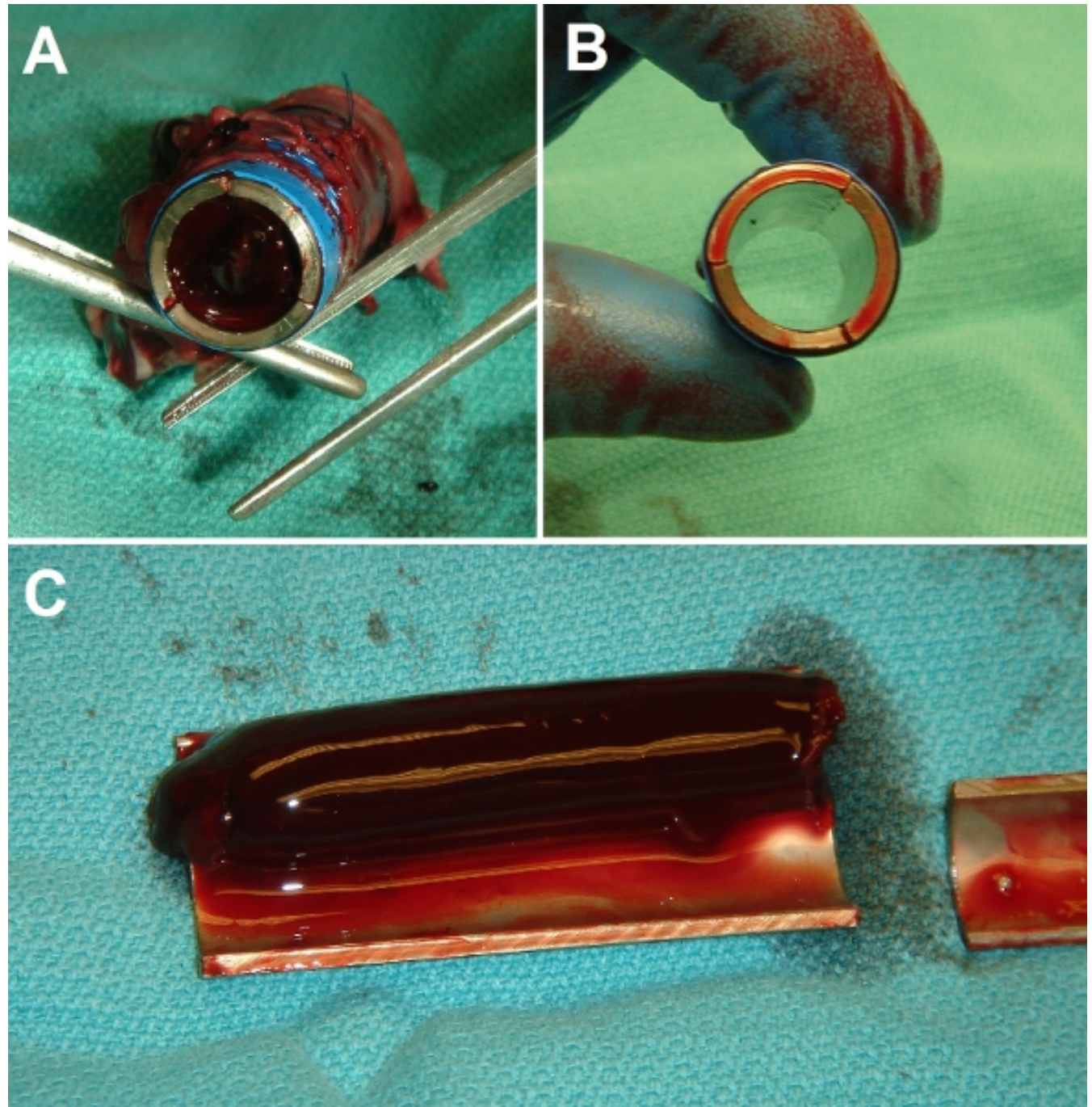

Figure 7. Representative gross results of implantation surgery. (A) End-view of control bare metal Ti tube after implantation in porcine inferior vena cava (IVC). Tube lumen is fully occluded with a solid clot. (B) End-view of EPC-seeded Ti tube after implantation. Tube lumen is fully patent and clear. (C) Dissected view of control (bare) Ti tube after 3 day implantation, showing extent of thrombosis (experiments were conducted up to 3 weeks duration with identical results).
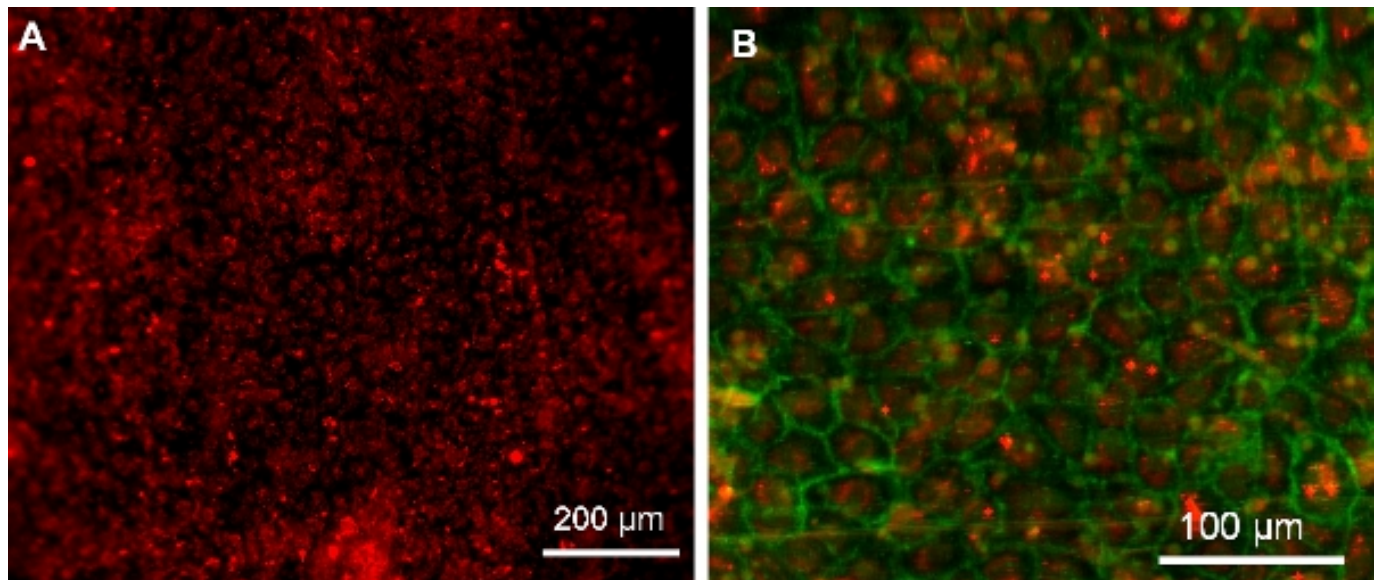

Figure 8. EPCs on Ti tube surface following 3 day implantation (imaged with an upright Leica DMRB microscope with a QImaging QICAM monochrome digital camera and Image Pro Plus software). (A) Confluent cells on surface showing PKH26 pre-surgery labeling. (B) Confluent layer of EPCs. Red: PKH26 pre-surgery labeling. Green: EPC PECAM-stain. 


\section{Discussion}

The method of cell-seeding Ti tubes presented here enables physicians and scientists to quickly and uniformly endothelialize blood-contacting surfaces of implantable devices. Since we isolate and expand EPCs from peripheral blood samples, no major invasive procedure is required to harvest these cells. Moreover, the EPCs are autologous; therefore, the risk of any immune reaction to the cell-seeded implant is eliminated. The principles demonstrated in his protocol can be utilized not only for Ti tubes, but for many other biomaterials, which are utilized in cardiovascular medicine.

Critical steps in this protocol are meticulous cleaning of the Ti tubes, as we found that any adherent film of contaminant compromises cell adherence. Further, a slow rotation speed (inversely proportional to the tube diameter) is essential during the seeding process, such that EPCs can slowly settle and adhere to the surface as the Ti tube is moving.

Our method of seeding immediately before implantation avoids impractical ex vivo culture times; cells adhere individually and later form a confluent sheet in vivo, avoiding the possibility of embolization as a sheet immediately following the re-establishment of flow. Our prior studies show that once the EPCs have grown to a confluent layer, they make an extra-cellular matrix to which they firmly adhere, additionally minimizing any possible sloughing of an endothelial sheet. Although the possibility of embolic detachment cannot be entirely ruled out, it seems to be multifold lower risk than thrombosis of the entire device surface, the problem that this therapy is designed to prevent.

Our approach of implantation into the low shear, prothrombotic environment of the inferior vena cava utilizes one of the most trusted large animal models for researching blood compatibility and thrombosis of devices ${ }^{5,6}$. Note that all animal care and experimentation was conducted in accordance with the National Institute of Health Guidelines for the Care and Use of Laboratory Animals and only after approval of the Duke University Institutional Animal Care and Use Committee.

In order to successfully utilize this implantation method to test biomaterials and devices, it is important to patiently skeletonize the IVC segment and ligate all venous branch vessels in preparation for device insertion, such that no bleeding around the device originates in a 'false lumen'. Another critical step is the addition of DPBS into the device lumen such that the cells on the inside tube surface remain moist during closure of the veinotomy and before reperfusion is initiated. If the device cannot be found in the location where it was implanted, it may have migrated 'upstream' in the IVC. This can be prevented by placing a suture (4-O Prolene) through the vein wall and through a $2-3 \mathrm{~mm}$ section of the PVC tubing so that the tube is firmly anchored in its present location. Should the researcher have difficulty finding the fluorescently pre-labeled cells shown in Figure 8 after explantation in an otherwise patent tube, it is likely that the cells have peeled off as a coherent sheet. This can be prevented by very gentle dissection of the surrounding vein and dis-assembly of the 3 Ti tube sections, after fixing the vein together with the tube.

Our technology provides proof-of-concept for preventing cardiovascular device thrombosis through EPC-seeding. This technology may be used in the development of 'biogenic' implants lined with patients' own endothelial progenitor cells. The feasibility study in our porcine animal model provides for the first steps toward translation of this 'personalized medicine' into clinical practice.

\section{Disclosures}

No conflicts of interest declared.

\section{Acknowledgements}

The authors would like to thank Leica Microsystems for their valuable advice on imaging titanium sections and Gemini Bio-Products for providing the porcine serum used in this study. We also like to thank the NIH for their support through Grant "Autologous EPC lining to improve biocompatibility of circulatory assist devices", RC1HL099863-01. Further, we are grateful for the National Science Foundation Graduate Research Fellowship's support of Alexandra Jantzen. We also like to thank George Quick, Mike Lowe and lanthia Parker for assisting with many aspects of the surgical procedure and handling of our research animals. Steven Owen has been invaluable in machining many parts of our seeding device and cutting titanium tubes.

\section{References}

1. Achneck, H.E., et al. Pathophysiology of bleeding and clotting in the cardiac surgery patient: from vascular endothelium to circulatory assist device surface. Circulation. 122, 2068-2077, doi:122/20/2068 [pii] 10.1161/CIRCULATIONAHA.110.936773 (2010).

2. Achneck, H.E., Sileshi, B., \& Lawson, J.H. Review of the biology of bleeding and clotting in the surgical patient. Vascular. 16 Suppl 1, S6-13 (2008).

3. Arvidsson, S., Askendal, A., \& Tengvall, P. Blood plasma contact activation on silicon, titanium and aluminium. Biomaterials. 28, 1346-1354, doi:S0142-9612(06)00951-3 [pii] 10.1016/j.biomaterials.2006.11.005 (2007).

4. Achneck, H.E., et al. The biocompatibility of titanium cardiovascular devices seeded with autologous blood-derived endothelial progenitor cells: EPC-seeded antithrombotic Ti Implants. Biomaterials. 32, 10-18, doi:S0142-9612(10)01111-7 [pii] 10.1016/j.biomaterials.2010.08.073 (2011).

5. Kang, C., Bonneau, M., Brouland, J.P., Bal dit Sollier, C., \& Drouet, L. In vivo pig models of venous thrombosis mimicking human disease. Thromb Haemost. 89, 256-263 (2003).

6. Ueberrueck, T., et al. Comparison of the ovine and porcine animal models for biocompatibility testing of vascular prostheses. $J$ Surg Res. 124, 305-311 (2005). 
7. Velik-Salchner, C., et al. Normal values for thrombelastography (ROTEM) and selected coagulation parameters in porcine blood. Thromb Res. 117, 597-602 (2006).

8. Yoder, M.C., et al. Redefining endothelial progenitor cells via clonal analysis and hematopoietic stem/progenitor cell principals. Blood. 109, 1801-1809 (2007).

9. Achneck, H.E., et al. in American Heart Association Scientific Sessions, Abstract Oral Sessions, Medical Aspects End Stage Heart Failure: Transplantation and Device Therapies. (2010).

10. Achneck, H.E., et al. Regenerating titanium ventricular assist device surfaces after gold/palladium coating for scanning electron microscopy. Microsc Res Tech. 73, 71-76, doi:10.1002/jemt.20757 (2010).

11. Ford, J.W., Welling, T.H., Stanley, J.C., \& Messina, L.M. PKH26 and 125I-PKH95: characterization and efficacy as labels for in vitro and in vivo endothelial cell localization and tracking. J Surg Res. 62, 23-28 (1996). 